Rico Berner, Simon Vock, Eckehard Schöll, Serhiy Yanchuk

\title{
Desynchronization Transitions in Adaptive Networks
}

Journal article | Accepted manuscript (Postprint)

This version is available at https://doi.org/10.14279/depositonce-11338

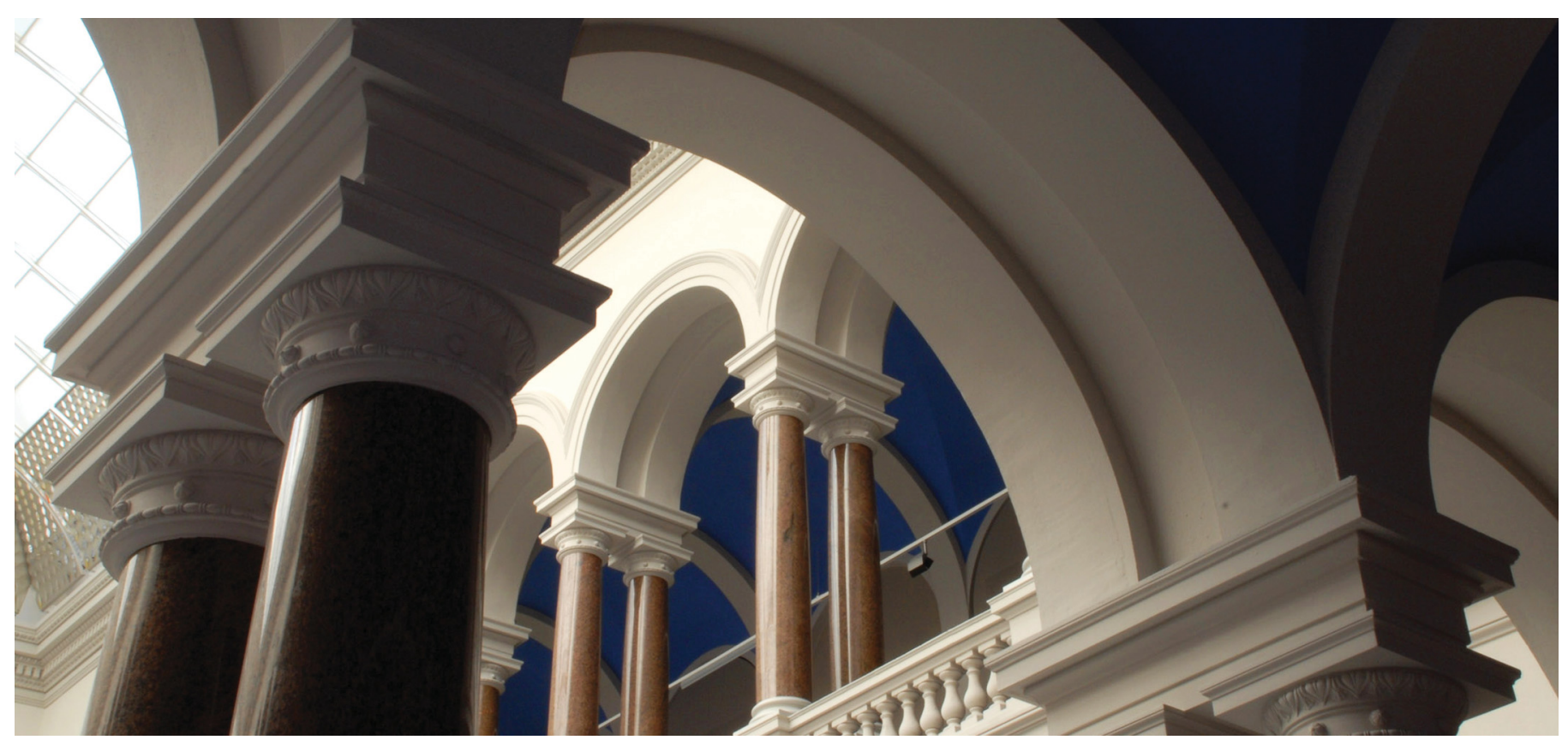

Berner, R., Vock, S., Schöll, E., \& Yanchuk, S. (2021). Desynchronization Transitions in Adaptive

Networks. Physical Review Letters, 126(2). https://doi.org/10.1103/physrevlett.126.028301

(c) 2021 American Physical Society 


\title{
Desynchronization transitions in adaptive networks
}

\author{
Rico Berner ${ }^{1,2},{ }^{*}$ Simon Vock $^{1}$, Eckehard Schöll ${ }^{1,3,4}$, and Serhiy Yanchuk ${ }^{2}$ \\ ${ }^{1}$ Institut für Theoretische Physik, Technische Universität Berlin, Hardenbergstr. 36, 10623 Berlin, Germany \\ ${ }^{2}$ Institut für Mathematik, Technische Universität Berlin, \\ Straße des 17. Juni 136, 10623 Berlin, Germany \\ ${ }^{3}$ Bernstein Center for Computational Neuroscience Berlin, \\ Humboldt-Universität, Philippstraße 13, 10115 Berlin, Germany and \\ ${ }^{4}$ Potsdam Institute for Climate Impact Research, Telegrafenberg A 31, 14473 Potsdam, Germany
}

(Dated: December 11, 2020)

\begin{abstract}
Adaptive networks change their connectivity with time, depending on their dynamical state. While synchronization in structurally static networks has been studied extensively, this problem is much more challenging for adaptive networks. In this Letter, we develop the master stability approach for a large class of adaptive networks. This approach allows for reducing the synchronization problem for adaptive networks to a low-dimensional system, by decoupling topological and dynamical properties. We show how the interplay between adaptivity and network structure gives rise to the formation of stability islands. Moreover, we report a desynchronization transition and the emergence of complex partial synchronization patterns induced by an increasing overall coupling strength. We illustrate our findings using adaptive networks of coupled phase oscillators and FitzHugh-Nagumo neurons with synaptic plasticity.
\end{abstract}

In nature and technology, complex networks serve as a ubiquitous paradigm with a broad range of applications from physics, chemistry, biology, neuroscience, socio-economic and other systems [1]. Dynamical networks are composed of interacting dynamical units, such as, e.g., neurons or lasers. Collective behavior in dynamical networks has attracted much attention over the last decades. Depending on the network and the specific dynamical system, various synchronization patterns of increasing complexity were explored [2-5]. Even in simple models of coupled oscillators, patterns such as complete synchronization [6], cluster synchronization [7-11], and various forms of partial synchronization have been found, such as frequency clusters [12], solitary [13] or chimera states [14-22]. In brain networks, particularly, synchronization is believed to play a crucial role: for instance, under normal conditions in the context of cognition and learning [23, 24], and under pathological conditions, such as Parkinson's disease [25], epilepsy [26-30], tinnitus [31, 32], schizophrenia, to name a few [33]. Also in power grid networks, synchronization is essential for the stable operation [34-37].

The powerful methodology of the master stability function [38] has been a milestone for the analysis of synchronization phenomena. This method allows for separating dynamical from structural features for a given dynamical network. It drastically simplifies the problem by reducing the dimension and unifying the synchronization study for different networks. Since its introduction, the master stability approach has been extended and refined for multilayer [39], multiplex [40, 41] and hypernetworks [42, 43]; to account for single and distributed delays [44-49]; and to describe the stability of clustered states [50-53]. The master stability function has been used to understand effects in temporal $[54,55]$ as well as adaptive networks [56] within a static formalism. Beyond the local stability described by the master stability function, Belykh et. al. have developed the connection graph stability method to provide analytic bounds for the global asymptotic stability of synchronized states [57-60]. Despite the apparent vivid interest in the stability features of synchronous states on complex networks, only little is known about the effects induced by an adaptive network structure. This lack of knowledge is even more surprising regarding how important adaptive networks are for the modeling of real-world systems.

Adaptive networks are commonly used models for synaptic plasticity [61-66] which determines learning, memory, and development in neural circuits. Moreover, adaptive networks have been reported for chemical [67, 68], epidemic [69], biological [70], transport [71], and social systems $[72,73]$. A paradigmatic example of adaptively coupled phase oscillators has recently attracted much attention [12, 41, 74-81], and it appears to be useful for predicting and describing phenomena in more realistic and detailed models [82-85]. Systems of phase oscillators are important for understanding synchronization phenomena in a wide range of applications [86-88].

In this Letter, we report on a surprising desynchronization transition induced by an adaptive network structure. We find various parameter regimes of partial synchronization during the transition from the synchronized to an incoherent state. The partial synchronization phenomena include multi-frequency-cluster and chimera-like states. By going beyond the static network paradigm, we develop a master stability approach for networks with adaptive coupling. We show how the adaptivity of the network gives rise to the emergence of stability islands in the master stability function that result in the desynchronization transition. With this, we establish a general framework to study those transitions for a wide range of 
dynamical systems. In order to provide analytic insights, we use the generalized Kuramoto-Sakaguchi system on an adaptive and complex network. Finally, we show that our findings also hold for a more realistic neuronal setup of coupled FitzHugh-Nagumo neurons with synaptic plasticity.

We consider the following general class of $N$ adaptively coupled systems [12, 41, 74-80, 89]

$$
\begin{aligned}
& \dot{\boldsymbol{x}}_{i}=f\left(\boldsymbol{x}_{i}\right)-\sigma \sum_{j=1}^{N} a_{i j} \kappa_{i j} g\left(\boldsymbol{x}_{i}, \boldsymbol{x}_{j}\right), \\
& \dot{\kappa}_{i j}=-\epsilon\left(\kappa_{i j}+a_{i j} h\left(\boldsymbol{x}_{i}-\boldsymbol{x}_{j}\right)\right),
\end{aligned}
$$

where $\boldsymbol{x}_{i} \in \mathbb{R}^{d}, i=1, \ldots, N$, is the $d$-dimensional dynamical variable of the $i$ th node, $f\left(\boldsymbol{x}_{i}\right)$ describes the local dynamics of each node, and $g\left(\boldsymbol{x}_{i}, \boldsymbol{x}_{j}\right)$ is the coupling function. The coupling is weighted by scalar variables $\kappa_{i j}$ which are adapted dynamically according to Eq. (2) with the nonlinear adaptation function $h\left(\boldsymbol{x}_{i}-\boldsymbol{x}_{j}\right)$. We assume that the adaptation depends on the difference of the corresponding dynamical variables, similar to the neuronal spike timing-dependent plasticity $[62,63,90,91]$. The base connectivity structure is given by the matrix elements $a_{i j} \in\{0,1\}$ of the $N \times N$ adjacency matrix $A$ which possesses a constant row sum $r$, i.e., $r=\sum_{j=1}^{N} a_{i j}$ for all $i=1, \ldots, N$. The assumption of the constant row sum is necessary to allow for synchronization. The Laplacian matrix is $L=r \mathbb{I}_{N}-A$ where $\mathbb{I}_{N}$ is the $N$-dimensional identity matrix. The eigenvalues of $L$ are called Laplacian eigenvalues of the network. The parameter $\sigma>0$ defines the overall coupling input, and $\epsilon>0$ is a timescale separation parameter. In particular, if the adaptation is slower than the local dynamics, the parameter $\epsilon$ is small.

Complete synchronization is defined by the $N-1$ constraints $\boldsymbol{x}_{1}=\boldsymbol{x}_{2}=\cdots=\boldsymbol{x}_{N}$. Denoting the synchronization state by $\boldsymbol{x}_{i}(t)=\boldsymbol{s}(t)$ and $\kappa_{i j}=\kappa_{i j}^{s}$, we obtain from Eqs. (1)-(2) the following equations for $s(t)$ and $\kappa_{i j}^{s}$

$$
\begin{aligned}
\dot{\boldsymbol{s}} & =f(\boldsymbol{s})+\sigma r h(0) g(\boldsymbol{s}, \boldsymbol{s}), \\
\kappa_{i j}^{s} & =-a_{i j} h(0) .
\end{aligned}
$$

In particular, we see that $s(t)$ satisfies the dynamical equation (3), and $\kappa_{i j}^{s}$ are either $-h(0)$ or zero, if the corresponding link in the base connectivity structure exists $\left(a_{i j}=1\right)$ or not $\left(a_{i j}=0\right)$, respectively.

To describe the local stability of the synchronous state, we introduce the variations $\boldsymbol{\xi}_{i}=\boldsymbol{x}_{i}-\boldsymbol{s}$ and $\chi_{i j}=\kappa_{i j}-$ $\kappa_{i j}^{s}$. The linearized equations for these variations can be written in a matrix form

$$
\left(\begin{array}{c}
\dot{\boldsymbol{\xi}} \\
\dot{\chi}
\end{array}\right)=\left(\begin{array}{cc}
S & -\sigma B \otimes g(\boldsymbol{s}, \boldsymbol{s}) \\
-\epsilon C \otimes \mathrm{D} h(0) & -\epsilon \mathbb{I}_{N^{2}}
\end{array}\right)\left(\begin{array}{c}
\boldsymbol{\xi} \\
\chi
\end{array}\right),
$$

where $\boldsymbol{\xi}=\left(\boldsymbol{\xi}_{1}^{T}, \ldots, \boldsymbol{\xi}_{N}^{T}\right)^{T}, \boldsymbol{\chi}=\left(\chi_{11}, \chi_{12}, \ldots, \chi_{N N}\right)^{T}$ are $N d$-dimensional and $N^{2}$-dimensional vectors, respec- tively,

$$
\begin{aligned}
S=\mathbb{I}_{N} \otimes & \mathrm{D} f(\boldsymbol{s}) \\
& +\sigma h(0)\left(r \mathbb{I}_{N} \otimes \mathrm{D}_{1} g(\boldsymbol{s}, \boldsymbol{s})+A \otimes \mathrm{D}_{2} g(\boldsymbol{s}, \boldsymbol{s})\right),
\end{aligned}
$$

$\mathrm{D} f$ and $\mathrm{D} h$ are the Jacobians $(d \times d$ matrix and $1 \times d$ matrix, respectively), $\mathrm{D}_{1} g$ and $\mathrm{D}_{2} g$ are the Jacobians with respect to the first and the second variable, respectively, and the constant matrices $B\left(N \times N^{2}\right)$ and $C\left(N^{2} \times N\right)$ are given in [106].

System (5) is used to calculate the Lyapunov exponents of the synchronous state; it possesses very high dimension $N^{2}+N d$. However, the Jacobian matrix in (5) is sparse with a large $N^{2} \times N^{2}$ block given by the simple diagonal matrix $-\epsilon \mathbb{I}_{N^{2}}$. This implies that (5) possess $N^{2}-N$ stable directions with Lyapunov exponents $-\epsilon$. To find these directions, we substitute $(\boldsymbol{\xi}, \boldsymbol{\eta})=e^{-\epsilon t}\left(\boldsymbol{\xi}_{0}, \boldsymbol{\eta}_{0}\right)$ into (5) and obtain the linear system

$$
\left(\begin{array}{cc}
S+\epsilon \mathbb{I}_{N d} & -\sigma B \otimes g(s, s) \\
-\epsilon C \otimes \operatorname{D} h(0) & 0
\end{array}\right)\left(\begin{array}{c}
\boldsymbol{\xi}_{0} \\
\chi_{0}
\end{array}\right)=0
$$

This system possesses at least $N^{2}-N$ linearly independent solutions, since the matrix in (6) is degenerate due to the large zero block [106].

Such a structure of the invariant subspaces in system (5) allows for introducing new coordinates, which separate the $N^{2}-N$ stable directions from the remaining $N(d+1)$ directions. With these new coordinates, we reduce the system's dimension significantly. Moreover, as in the classical master stability approach, we diagonalize the $N(d+1)$-dimensional master system into blocks of $d+1$ dimensions. Hence, the dynamics in each block is described by the new coordinates $\zeta$ and $\kappa$ which are $d$ - and one-dimensional dynamical variables, respectively. For further details and the proof of the master stability function, we refer to the Supplemental Material [106]. Our analysis shows that the coupling structure enters just as a complex parameter $\mu$, the network's Laplacian eigenvalue.

As a result, the stability problem is reduced to the largest Lyapunov exponent $\Lambda(\mu)$, depending on a complex parameter $\mu$, for the following system

$$
\begin{aligned}
\dot{\boldsymbol{\zeta}}=( & \mathrm{D} f(\boldsymbol{s})+\sigma r h(0)\left[\mathrm{D}_{1} g(\boldsymbol{s}, \boldsymbol{s})\right. \\
& \left.\left.\quad+\left(1-\frac{\mu}{r}\right) \mathrm{D}_{2} g(\boldsymbol{s}, \boldsymbol{s})\right]\right) \boldsymbol{\zeta}-\sigma g(\boldsymbol{s}, \boldsymbol{s}) \kappa, \\
\dot{\kappa}=- & \epsilon(\mu \mathrm{D} h(0) \boldsymbol{\zeta}+\kappa) .
\end{aligned}
$$

The function $\Lambda(\mu)$ is called master stability function. Note that the first bracketed term in $\boldsymbol{\zeta}$ of (7) resembles the master stability approach for static networks, which, in this case, is equipped by an additional interaction representing the adaptation. Furthermore, the shape of the master stability function depends on the 
choice of $\sigma$ and $r$ explicitly. In case of diffusive coupling, i.e., $g(\boldsymbol{x}, \boldsymbol{y})=g(\boldsymbol{x}-\boldsymbol{y})$, the master stability function can be expressed as $\Lambda(\sigma \mu)$ such that the shape of $\Lambda$ scales linearly with the coupling constant $\sigma$.

To obtain analytic insights into the stability features of synchronous states that are induced by an adaptive coupling structure, we consider the following model of $N$ adaptively coupled phase oscillators $[12,76]$

$$
\begin{gathered}
\dot{\phi}_{i}=\omega-\sigma \sum_{j=1}^{N} a_{i j} \kappa_{i j} \sin \left(\phi_{i}-\phi_{j}+\alpha\right), \\
\dot{\kappa}_{i j}=-\epsilon\left(\kappa_{i j}+a_{i j} \sin \left(\phi_{i}-\phi_{j}+\beta\right)\right),
\end{gathered}
$$

where $\phi_{i}$ represents the phase of the $i$ th oscillator, $\omega$ is its natural frequency which we set to zero in a rotating frame. The phase-lag $\alpha$ can be regarded as propagation delay in the context of neuronal systems [92].

The synchronous state of $(9)-(10)$ is given by $\boldsymbol{s}(t)=$ $(\sigma r \sin \alpha \sin \beta) t$ and $\kappa_{i j}^{s}=-a_{i j} \sin \beta$. Using (7)-(8), the stability of the synchronous state is described by the quadratic characteristic polynomial

$$
\lambda^{2}+(\epsilon-\sigma \mu \cos (\alpha) \sin (\beta)) \lambda-\epsilon \sigma \mu \sin (\alpha+\beta)=0 .
$$

The master stability function for the synchronous solution is given as the maximum real part $\Lambda=\max \operatorname{Re}\left(\lambda_{1,2}\right)$ of the solutions $\lambda_{1,2}$ of the polynomial (11). These solutions $\lambda_{1,2}$ should be considered as functions of the complex parameter $\mu$ determining the network structure. It is convenient, however, to use the parameter $\sigma \mu$ in our case.

Figure 1 displays the master stability function determined for different adaptation rules controlled by $\beta$. The blue-colored areas correspond to regions that lead to stable dynamics. By changing the control parameter $\beta$, various shapes of the stable regions are visible. For some parameters, e.g., Fig. 1(c,d,e), almost a whole half-space either left or right of the imaginary axis belongs to the stable regime. This resembles the case of no adaptation where the stability of the synchronous state is solely described by the sign of the real part of $\sigma \mu \sin \beta \cos \alpha$, see Fig. 1(a,b). Note that in the case of no adaptation $(\epsilon=0)$ there exist $N^{2}$ neutral directions with zero eigenvalues that do not affect the stability, and correspond to the variations of the coupling weights. We also find parameters where most values $\sigma \mu$ correspond to unstable dynamics, except for an island, i.e., a bounded region in $\sigma \mu$ parameter space, see Fig. 1(f).

To understand the emergence of the stability islands, we analyze the boundary that separates the stable $(\Lambda<$ $0)$ from the unstable region $(\Lambda>0)$. This boundary is given by the condition $\Lambda=\operatorname{Re} \lambda=0$, or, equivalently, $\lambda=\mathrm{i} \gamma$. Substituting this into Eq. (11), we obtain a parameterized expression for the boundary as a function of $\gamma$ that has the form $\sigma \mu=Z(\gamma)$ with $Z(\gamma)$ given explicitly in the Supplemental material [106]. The latter

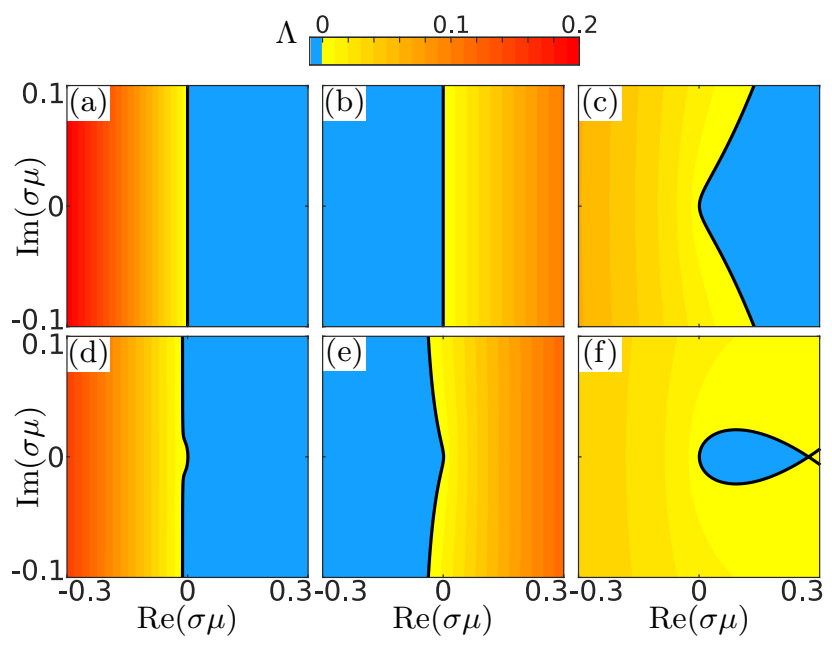

FIG. 1. Master stability function $\Lambda(\sigma \mu)$ for the adaptive phase oscillator network (9)-(10). Regions belonging to negative Lyapunov exponents $\Lambda$ are colored blue. The curve where $\Lambda(\mu)=0$ is given as a black solid line. In panels (a) and (b) the case without adaptation $(\epsilon=0)$ is presented for $\beta=-0.35 \pi$ and $\beta=0.2 \pi$, respectively. Other panels: $\epsilon=0.01$ and (c) $\beta=-0.95 \pi$, (d) $\beta=-0.35 \pi$, (e) $\beta=0.2 \pi$, and (f) $\beta=0.98 \pi$. In all panels $\alpha=0.3 \pi$.

parametrization of the boundary is displayed in Fig. 1 as the solid black line. It is straightforward to show that a stability island exists if $\sin (\alpha+\beta) /(\cos \alpha \sin \beta)<0$. The latter condition indicates a certain balance between the coupling and adaptation function. We emphasize that the emergence of stability islands is a direct consequence of adaptation. Without adaptation, the boundary simplifies to the axis $\operatorname{Re} \mu=0$, see Figs. 1(a,b). Intuitively, the presence of adaptivity, i.e., Eq. (8), provides a feedback mechanism that can change the stability (e.g., by an additional effective phase lag), and hence gives rise to the emergence of stability islands of the master stability function.

In the following, we analyze the behavior of the adaptive network of phase oscillators (9)-(10) in the presence of a stability island, and show how such an island introduces a desynchronization transition with increasing overall coupling $\sigma$. To measure the coherence, we use the cluster parameter $R_{C}[76,79]$, which is given by the number of pairwise coherent oscillators normalized by the total number of pairs $N^{2}$. In the case of complete synchronization, frequency clustering, or incoherence, the cluster parameter values are $R_{C}=1,1<R_{C}<0$, or $R_{C}=0$, respectively, see Supplemental Material for details [106].

The top panel in Fig. 2 shows the cluster parameter $R_{C}$ for different values of the overall coupling constant $\sigma$. We observe that for small $\sigma$, the synchronous state is stable, see Fig. 2(a,d,g). This stability follows directly from the master stability function since all values $\sigma \mu_{i}$ for all Laplacian eigenvalues lie within the stability island, see Fig. 2(a). 

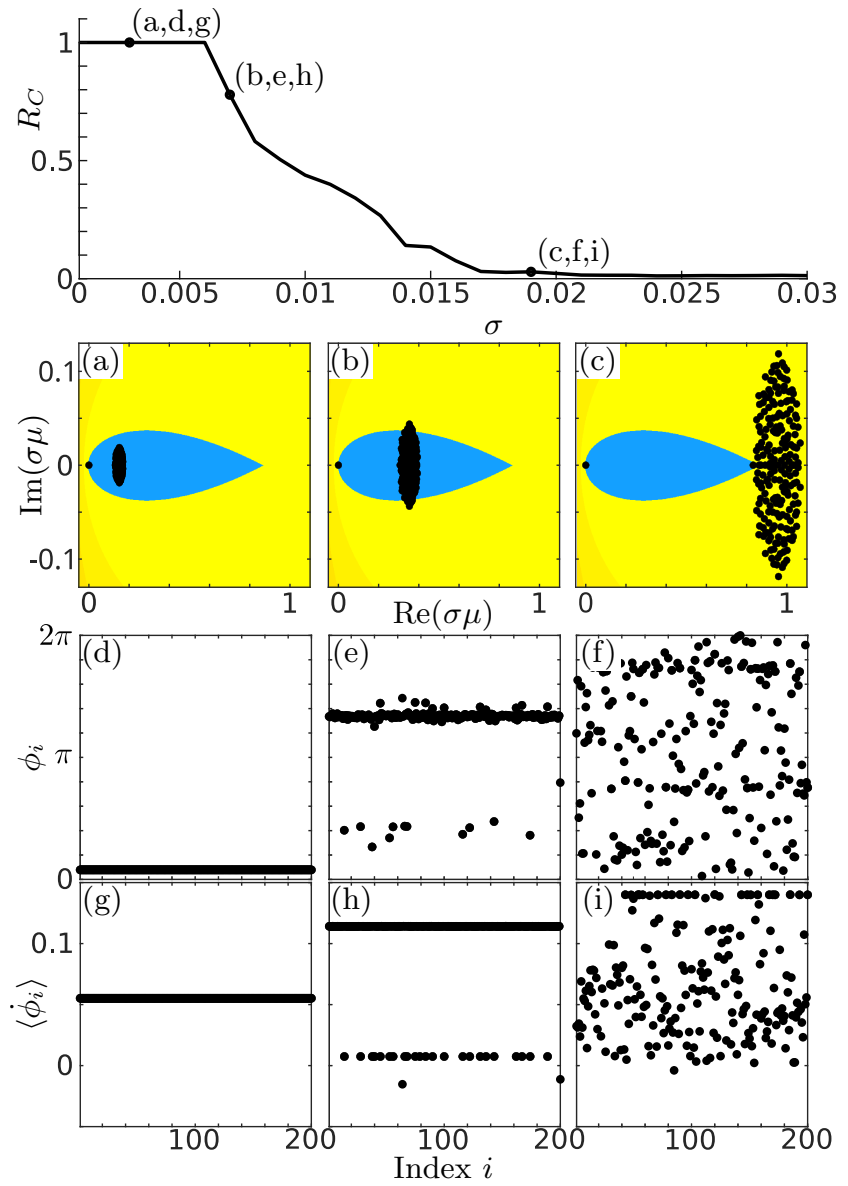

FIG. 2. Dynamics in the network of 200 oscillators (9)-(10) with random adjacency matrix $A_{\mathrm{c}}$ [106], and different values of overall coupling strength $\sigma$. Adiabatic continuation for increasing $\sigma$ with the stepsize of 0.001 , starting with the synchronous state $\phi_{i}=0, \kappa_{i j}=-a_{i j} \sin \beta$. The top panel shows the cluster parameter $R_{C}$ vs $\sigma$. For the three values of $\sigma$ : $(\mathrm{a}, \mathrm{d}, \mathrm{g}) \sigma=0.003$, (b,e,h) $\sigma=0.007$, and (c,f,i) $\sigma=0.019$, the plots show: in $(\mathrm{a}, \mathrm{b}, \mathrm{c})$ the master stability function color coded as in Fig. 1, together with $\sigma \mu_{i}$, where $\mu_{i}$ are the $N$ Laplacian eigenvalues of $A_{c}$; in (d,e,f) snapshots for $\phi_{i}$ at $t=30000$; and in $(\mathrm{g}, \mathrm{h}, \mathrm{i})$ the temporal average of the phase velocities $\left\langle\dot{\phi}_{i}\right\rangle$ over the last 5000 time units. Other parameters: $\alpha=0.49 \pi, \beta=0.88 \pi, \epsilon=0.01$.

By increasing the coupling strength $\sigma$, the values $\sigma \mu_{i}$ move out of the stability island ( $\mu_{i}$ remain the same), and the synchronous state becomes unstable, see Fig. 2(b,c). For intermediate values of $\sigma$, multiclusters with hierarchical structure in the cluster size emerge, see Fig. 2(e,h) for a three-cluster state. Increasing the coupling constant further leads to the emergence of incoherence. In Fig. 2(f,i), the coexistence of a coherent and an incoherent cluster is presented. Such chimera-like states have been numerically studied for adaptive networks in $[76,78,79]$.

In the following, we show how our findings are transferred to a more realistic set-up of coupled neurons with synaptic plasticity. For this, we consider a network of FitzHugh-Nagumo neurons [93-96] coupled through chemical excitatory synapses [97-99] equipped with plasticity. The form of the synaptic plasticity is similar to the rules used in $[84,100]$, with control parameters $\beta_{1}$ and $\beta_{2}$ of the adaptation function which are uniquely determined by the values of $h(0)$ and $\mathrm{D} h(0)$ of the plasticity rule, and these are the only essential parameters of the plasticity function, regarding the stability of the synchronous state, see Eqs. (7)-(8). For more details on the model, we refer to $[99,106]$.

The synchronous state of the network of FitzHughNagumo neurons satisfies Eqs. (3)-(4), and it is periodic for the chosen parameter values. Using our extended master stability approach, we determine numerically the master stability function which is the maximum Lyapunov exponent of Eqs. (7)-(8).

In Fig. 3(a,b,c), we show the master stability function in dependence on the parameter $\mu / r$ for different values of the overall coupling constant $\sigma$. We observe a stability island for the chosen set of parameters, see the Supplemental material for other parameter values [106]. In contrast to the phase oscillator network in Fig. 2, the shape of the master stability function does not scale linearly with $\sigma$. This is due to the non-diffusive coupling function, see [106] for details. Moreover, with increasing $\sigma$, the size of the stability island shrinks. Since all Laplacian eigenvalues $\mu_{i}$ are independent of $\sigma$, we observe that $\mu_{i} / r$ move out of the stability island with increasing $\sigma$. For the globally coupled network, in particular, we have either $\mu_{i} / r=0$ or $\mu_{i} / r=1$. Therefore, with increasing $\sigma$, we find a transition from complete coherence, see Fig. 3(a,d,g) to partial synchronization and incoherence. We further observe that closely after destabilization, a large frequency cluster remains visible, see Fig. 3(b,e,h). For higher overall coupling, the cluster sizes shrink, and the number of small clusters increases, see Fig. 3(c,f,i).

In summary, we have developed a master stability approach for a general class of adaptive networks. This approach allows for studying the subtle interplay between nodal dynamics, adaptivity, and a complex network structure. The master stability approach has been first applied to a paradigmatic model of adaptively coupled phase oscillators. We have presented several typical forms of the master stability function for different adaptation rules, and observed adaptivity-induced stability islands. Besides, we have shown that stability islands give rise to the emergence of multicluster states and chimera-like states in the desynchronization transition for an increasing overall coupling strength. Qualitatively the same phenomena have been shown for a more realistic network of non-diffusively coupled FitzHugh-Nagumo neurons with synaptic plasticity. In this set-up, the emergence of a stability island and a desynchronization transition have been found as well.

The theoretical approach introduced in this Letter pro- 

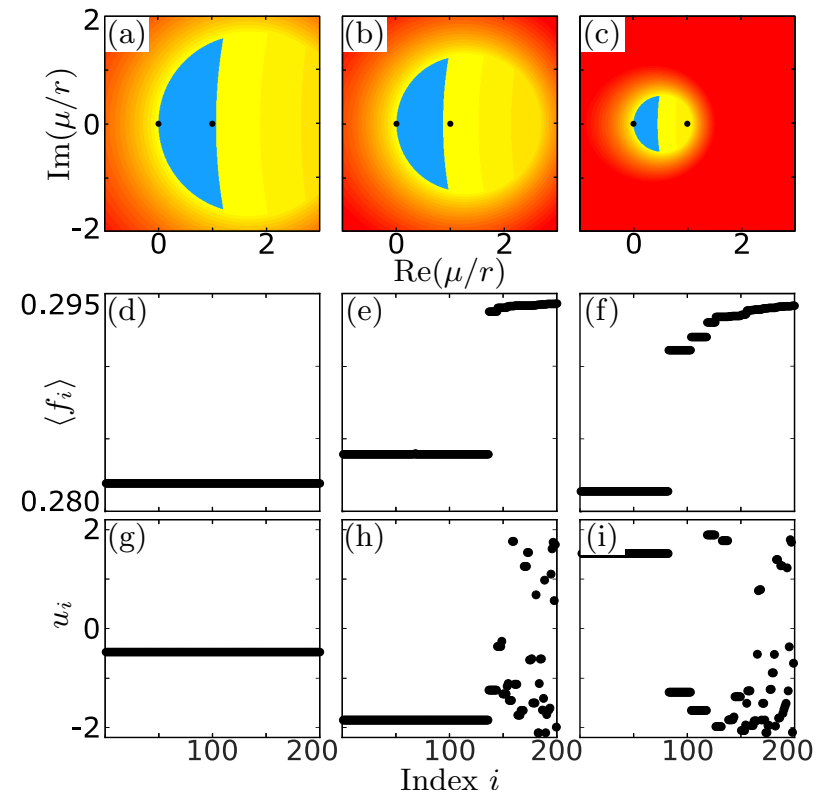

FIG. 3. Dynamics of globally coupled network of 200 FitzHugh-Nagumo neurons with plasticity, see [106] for details. Adiabatic continuation for an increasing overall coupling strength $\sigma$ with the step size 0.0005 , starting with the synchronous state. For the three values of $\sigma$ : (a,d,g) $\sigma=0.002$, (b,e,h) $\sigma=0.0025$, and (c,f,i) $\sigma=0.005$, the plots show: in (a,b,c), the master stability function, together with $\mu_{i} / r$, where $\mu_{i}$ are the Laplacian eigenvalues (color code as in Fig. 1), in (d,e,f) the average frequency $\left\langle f_{i}\right\rangle$, and in $(\mathrm{g}, \mathrm{h}, \mathrm{i})$ snapshots for the membrane potential $u_{i}$ at $t=10000$. Here $\left\langle f_{i}\right\rangle=M_{i} / 1000$, where $M_{i}$ is the number of rotations (spikes) of neuron $i$ during the time interval of length 1000 . The control parameters for the adaptation rule $\beta_{1}$ and $\beta_{2}$ are chosen such that $h(0)=0.8$ and $\operatorname{Dh}(0)=(80,0,0)$ for the adaptation function $h$.

vides a powerful tool to study collective effects in more realistic neuronal network models, including synaptic plasticity $[32,82]$. While our approach is presented for differentiable models, it might be generalized to noncontinuous models of spiking neurons equipped with spike timing-dependent plasticity [90, 91]. Our generalized master stability approach relies on a reduction method that depends only on the network structure. Therefore, the method allows for extensions to systems with single or even distributed delays [47, 48] as they are of crucial importance in neuronal circuits. Our findings on the transition from coherence to incoherence reveal the role adaptivity plays for the formation of partially synchronized patterns which are important for understanding the functioning of neuronal systems [101]. Beyond neuronal networks, adaptation is a well-known control paradigm [102-105]. Our extended master stability approach provides a generalized framework to study various adaptive control schemes for a wide range of dynamical systems.

This work was supported by the German Re- search Foundation DFG, Project Nos. 411803875 and 440145547.

* rico.berner@physik.tu-berlin.de

[1] M. E. J. Newman: The structure and function of complex networks, SIAM Review 45, 167 (2003).

[2] A. Pikovsky, M. Rosenblum, and J. Kurths: Synchronization: a universal concept in nonlinear sciences (Cambridge University Press, Cambridge, 2001), 1st ed.

[3] S. H. Strogatz: Exploring complex networks, Nature 410, 268 (2001).

[4] A. Arenas, A. Díaz-Guilera, J. Kurths, Y. Moreno, and C. Zhou: Synchronization in complex networks, Phys. Rep. 469, 93 (2008).

[5] S. Boccaletti, A. N. Pisarchik, C. I. del Genio, and A. Amann: Synchronization: From Coupled Systems to Complex Networks (Cambridge University Press, Cambridge, 2018).

[6] Y. Kuramoto: Chemical Oscillations, Waves and Turbulence (Springer-Verlag, Berlin, 1984).

[7] S. Yanchuk, Y. Maistrenko, and E. Mosekilde: Partial synchronization and clustering in a system of diffusively coupled chaotic oscillators, Math. Comp. Simul. 54, 491 (2001).

[8] F. Sorrentino and E. Ott: Network synchronization of groups, Phys. Rev. E 76, 056114 (2007).

[9] I. Belykh and M. Hasler: Mesoscale and clusters of synchrony in networks of bursting neurons, Chaos 21, 016106 (2011).

[10] M. Golubitsky and I. Stewart: Rigid patterns of synchrony for equilibria and periodic cycles in network $d y$ namics, Chaos 26, 094803 (2016).

[11] Y. Zhang and A. E. Motter: Symmetry-independent stability analysis of synchronization patterns, SIAM Rev. 62, 817 (2020).

[12] R. Berner, E. Schöll, and S. Yanchuk: Multiclusters in networks of adaptively coupled phase oscillators, SIAM J. Appl. Dyn. Syst. 18, 2227 (2019).

[13] P. Jaros, S. Brezetsky, R. Levchenko, D. Dudkowski, T. Kapitaniak, and Y. Maistrenko: Solitary states for coupled oscillators with inertia, Chaos 28, 011103 (2018).

[14] Y. Kuramoto and D. Battogtokh: Coexistence of Coherence and Incoherence in Nonlocally Coupled Phase Oscillators., Nonlin. Phen. in Complex Sys. 5, 380 (2002).

[15] D. M. Abrams and S. H. Strogatz: Chimera states for coupled oscillators, Phys. Rev. Lett. 93, 174102 (2004).

[16] A. E. Motter: Nonlinear dynamics: Spontaneous synchrony breaking, Nat. Phys. 6, 164 (2010).

[17] M. J. Panaggio and D. M. Abrams: Chimera states: Coexistence of coherence and incoherence in networks of coupled oscillators, Nonlinearity 28, R67 (2015).

[18] E. Schöll: Synchronization patterns and chimera states in complex networks: interplay of topology and dynamics, Eur. Phys. J. Spec. Top. 225, 891 (2016).

[19] S. Majhi, B. K. Bera, D. Ghosh, and M. Perc: Chimera states in neuronal networks: A review, Phys. Life Rev. 26, 100 (2019).

[20] O. E. Omel'chenko and E. Knobloch: Chimerapedia: coherence-incoherence patterns in one, two and three 
dimensions, New J. Phys. 21, 093034 (2019).

[21] E. Schöll, A. Zakharova, and R. G. Andrzejak: Editorial on the research topic: Chimera states in complex networks, Front. Appl. Math. Stat. 5, 62 (2019), doi: 10.3389/fams.2019.00062.

[22] A. Zakharova: Chimera Patterns in Networks: Interplay between Dynamics, Structure, Noise, and Delay, Understanding Complex Systems (Springer, 2020).

[23] W. Singer: Neuronal Synchrony: A Versatile Code Review for the Definition of Relations?, Neuron 24, 49 (1999).

[24] J. Fell and N. Axmacher: The role of phase synchronization in memory processes, Nat. Rev. Neurosci. 12, 105 (2011).

[25] C. Hammond, H. Bergman, and P. Brown: Pathological synchronization in Parkinson's disease: networks, models and treatments, Trends Neurosci. 30, 357 (2007).

[26] P. Jiruska, M. de Curtis, J. G. R. Jefferys, C. A. Schevon, S. J. Schiff, and K. Schindler: Synchronization and desynchronization in epilepsy: controversies and hypotheses, J. Physiol. 591.4, 787 (2013).

[27] V. K. Jirsa, W. C. Stacey, P. P. Quilichini, A. I. Ivanov, and C. Bernard: On the nature of seizure dynamics, Brain 137, 2210 (2014).

[28] A. Rothkegel and K. Lehnertz: Irregular macroscopic dynamics due to chimera states in small-world networks of pulse-coupled oscillators, New J. Phys. 16, 055006 (2014).

[29] R. G. Andrzejak, C. Rummel, F. Mormann, and K. Schindler: All together now: Analogies between chimera state collapses and epileptic seizures, Sci. Rep. 6, 23000 (2016).

[30] M. Gerster, R. Berner, J. Sawicki, A. Zakharova, A. Skoch, J. Hlinka, K. Lehnertz, and E. Schöll: FitzHugh-Nagumo oscillators on complex networks mimic epileptic-seizure-related synchronization phenomena, Chaos p. in print (2020), arXiv:2007.05497, Editor's Pick.

[31] P. A. Tass, I. Adamchic, H. J. Freund, T. von Stackelberg, and C. Hauptmann: Counteracting tinnitus by acoustic coordinated reset neuromodulation, Restor. Neurol. Neurosci. 30, 137 (2012).

[32] P. A. Tass and O. V. Popovych: Unlearning tinnitusrelated cerebral synchrony with acoustic coordinated reset stimulation: theoretical concept and modelling, Biol. Cybern. 106, 27 (2012).

[33] P. Uhlhaas, G. Pipa, B. Lima, L. Melloni, S. Neuenschwander, D. Nikolic, and W. Singer: Neural synchrony in cortical networks: history, concept and current status, Front. Integr. Neurosci. 3, 17 (2009).

[34] M. Rohden, A. Sorge, M. Timme, and D. Witthaut: Self-organized synchronization in decentralized power grids, Phys. Rev. Lett. 109, 064101 (2012).

[35] A. E. Motter, S. A. Myers, M. Anghel, and T. Nishikawa: Spontaneous synchrony in power-grid networks, Nat. Phys. 9, 191 (2013).

[36] P. J. Menck, J. Heitzig, J. Kurths, and H. J. Schellnhuber: How dead ends undermine power grid stability, Nat. Commun. 5, 3969 (2014).

[37] H. Taher, S. Olmi, and E. Schöll: Enhancing power grid synchronization and stability through time delayed feedback control, Phys. Rev. E 100, 062306 (2019).

[38] L. M. Pecora and T. L. Carroll: Master Stability Functions for Synchronized Coupled Systems, Phys. Rev.
Lett. 80, 2109 (1998).

[39] A. Brechtel, P. Gramlich, D. Ritterskamp, B. Drossel, and T. Gross: Master stability functions reveal diffusion-driven pattern formation in networks, Phys. Rev. E 97, 032307 (2018).

[40] L. Tang, X. Wu, J. Lü, J. Lu, and R. M. D'Souza: Master stability functions for complete, intralayer, and interlayer synchronization in multiplex networks of coupled rössler oscillators, Phys. Rev. E 99 (2019), 012304.

[41] R. Berner, J. Sawicki, and E. Schöll: Birth and stabilization of phase clusters by multiplexing of adaptive networks, Phys. Rev. Lett. 124, 088301 (2020).

[42] F. Sorrentino: Synchronization of hypernetworks of coupled dynamical systems, New J. Phys. 14, 033035 (2012).

[43] R. Mulas, C. Kuehn, and J. Jost: Coupled dynamics on hypergraphs: Master stability of steady states and synchronization, Phys. Rev. E 101, 062313 (2020).

[44] C. U. Choe, T. Dahms, P. Hövel, and E. Schöll: Controlling synchrony by delay coupling in networks: from in-phase to splay and cluster states, Phys. Rev. E 81, 025205(R) (2010).

[45] V. Flunkert, S. Yanchuk, T. Dahms, and E. Schöll: Synchronizing distant nodes: a universal classification of networks, Phys. Rev. Lett. 105, 254101 (2010).

[46] A. Keane, T. Dahms, J. Lehnert, S. A. Suryanarayana, P. Hövel, and E. Schöll: Synchronisation in networks of delay-coupled type-I excitable systems, Eur. Phys. J. B 85, 407 (2012).

[47] Y. N. Kyrychko, K. B. Blyuss, and E. Schöll: Synchronization of networks of oscillators with distributed-delay coupling, Chaos 24, 043117 (2014).

[48] J. Lehnert: Controlling synchronization patterns in complex networks, Springer Theses (Springer, Heidelberg, 2016).

[49] R. Börner, P. Schultz, B. Ünzelmann, D. Wang, F. Hellmann, and J. Kurths: Delay master stability of inertial oscillator networks, Phys. Rev. Research 2, 023409 (2020)

[50] T. Dahms, J. Lehnert, and E. Schöll: Cluster and group synchronization in delay-coupled networks, Phys. Rev. E 86, 016202 (2012).

[51] L. M. Pecora, F. Sorrentino, A. M. Hagerstrom, T. E. Murphy, and R. Roy: Symmetries, cluster synchronization, and isolated desynchronization in complex networks, Nat. Commun. 5, 4079 (2014).

[52] C. I. del Genio, J. Gómez-Gardeñes, I. Bonamassa, and S. Boccaletti: Synchronization in networks with multiple interaction layers, Sci. Adv. 2, e1601679 (2016).

[53] K. A. Blaha, K. Huang, F. Della Rossa, L. M. Pecora, M. Hossein-Zadeh, and F. Sorrentino: Cluster synchronization in multilayer networks: A fully analog experiment with lc oscillators with physically dissimilar coupling, Phys. Rev. Lett. 122, 014101 (2019).

[54] D. J. Stilwell, E. M. Bollt, and D. G. Roberson: Sufficient conditions for fast switching synchronization in time-varying network topologies, SIAM J. Appl. Dyn. Syst. 5, 140 (2006).

[55] V. Kohar, P. Ji, A. Choudhary, S. Sinha, and J. Kurths: Synchronization in time-varying networks, Phys. Rev. E 90, 022812 (2014).

[56] C. Zhou and J. Kurths: Dynamical weights and enhanced synchronization in adaptive complex networks, 
Phys. Rev. Lett. 96, 164102 (2006).

[57] V. N. Belykh, I. Belykh, and M. Hasler: Connection graph stability method for synchronized coupled chaotic systems, Physica D 195, 159 (2004).

[58] I. Belykh, E. de Lange, and M. Hasler: Synchronization of bursting neurons: What matters in the network topology, Phys. Rev. Lett. 94, 188101 (2005).

[59] I. Belykh, V. N. Belykh, and M. Hasler: Generalized connection graph method for synchronization in asymmetrical networks, Physica D 224, 42 (2006).

[60] I. Belykh, V. N. Belykh, and M. Hasler: Synchronization in asymmetrically coupled networks with node balance, Chaos 16, 015102 (2006).

[61] H. Markram, J. Lübke, M. Frotscher, and B. Sakmann: Regulation of synaptic efficacy by coincidence of postsynaptic APs and EPSPs., Science 275, 213 (1997).

[62] L. F. Abbott and S. Nelson: Synaptic plasticity: taming the beast, Nat. Neurosci. 3, 1178 (2000).

[63] N. Caporale and Y. Dan: Spike timing-dependent plasticity: A Hebbian learning rule, Annu. Rev. Neurosci. 31, 25 (2008).

[64] C. Meisel and T. Gross: Adaptive self-organization in a realistic neural network model, Phys. Rev. E 80, 061917 (2009).

[65] K. Mikkelsen, A. Imparato, and A. Torcini: Emergence of slow collective oscillations in neural networks with spike-timing dependent plasticity, Phys. Rev. Lett. 110, 208101 (2013).

[66] K. Mikkelsen, A. Imparato, and A. Torcini: Sisyphus effect in pulse-coupled excitatory neural networks with spike-timing-dependent plasticity, Phys. Rev. E 89, 062701 (2014).

[67] S. Jain and S. Krishna: A model for the emergence of cooperation, interdependence, and structure in evolving networks, Proc. Natl. Acad. Sci. 98, 543 (2001).

[68] C. Kuehn: Multiscale dynamics of an adaptive catalytic network, Math. Model. Nat. Phenom. 14, 402 (2019).

[69] T. Gross, C. J. D. D'Lima, and B. Blasius: Epidemic dynamics on an adaptive network, Phys. Rev. Lett. 96, 208701 (2006).

[70] S. R. Proulx, D. E. L. Promislow, and P. C. Phillips: Network thinking in ecology and evolution, Trends Ecol. Evol. 20, 345 (2005).

[71] E. A. Martens and K. Klemm: Transitions from trees to cycles in adaptive flow networks, Front. Phys. 5, 62 (2017).

[72] T. Gross and B. Blasius: Adaptive coevolutionary networks: a review, J. R. Soc. Interface 5, 259 (2008).

[73] L. Horstmeyer and C. Kuehn: Adaptive voter model on simplicial complexes, Phys. Rev. E 101, 022305 (2020).

[74] R. Gutiérrez, A. Amann, S. Assenza, J. GómezGardeñes, V. Latora, and S. Boccaletti: Emerging meso- and macroscales from synchronization of adaptive networks, Phys. Rev. Lett. 107, 234103 (2011).

[75] X. Zhang, S. Boccaletti, S. Guan, and Z. Liu: Explosive synchronization in adaptive and multilayer networks, Phys. Rev. Lett. 114, 038701 (2015).

[76] D. V. Kasatkin, S. Yanchuk, E. Schöll, and V. I. Nekorkin: Self-organized emergence of multi-layer structure and chimera states in dynamical networks with adaptive couplings, Phys. Rev. E 96, 062211 (2017).

[77] M. M. Asl, A. Valizadeh, and P. A. Tass: Dendritic and axonal propagation delays may shape neuronal networks with plastic synapses, Front. Physiol. 9, 1849 (2018).
[78] D. V. Kasatkin and V. I. Nekorkin: Synchronization of chimera states in a multiplex system of phase oscillators with adaptive couplings, Chaos 28, 093115 (2018).

[79] D. V. Kasatkin and V. I. Nekorkin: The effect of topology on organization of synchronous behavior in dynamical networks with adaptive couplings, Eur. Phys. J. Spec. Top. 227, 1051 (2018).

[80] R. Berner, J. Fialkowski, D. V. Kasatkin, V. I. Nekorkin, S. Yanchuk, and E. Schöll: Hierarchical frequency clusters in adaptive networks of phase oscillators, Chaos 29, 103134 (2019).

[81] P. Feketa, A. Schaum, and T. Meurer: Synchronization and multi-cluster capabilities of oscillatory networks with adaptive coupling, IEEE Trans. Autom. Control (2019).

[82] O. V. Popovych, M. N. Xenakis, and P. A. Tass: The spacing principle for unlearning abnormal neuronal synchrony, PLoS ONE 10, e0117205 (2015).

[83] L. Lücken, O. V. Popovych, P. Tass, and S. Yanchuk: Noise-enhanced coupling between two oscillators with long-term plasticity, Phys. Rev. E 93, 032210 (2016).

[84] S. Chakravartula, P. Indic, B. Sundaram, and T. Killingback: Emergence of local synchronization in neuronal networks with adaptive couplings, PLoS ONE 12, e0178975 (2017).

[85] V. Röhr, R. Berner, E. L. Lameu, O. V. Popovych, and S. Yanchuk: Frequency cluster formation and slow oscillations in neural populations with plasticity, PLoS ONE 14, e0225094 (2019).

[86] M. Breakspear, S. Heitmann, and A. Daffertshofer: Generative models of cortical oscillations: neurobiological implications of the Kuramoto model, Front. Hum. Neurosci. 4, 190 (2010).

[87] A. Nabi and J. Moehlis: Single input optimal control for globally coupled neuron networks, J. Neural Eng. 8, 065008 (2011).

[88] C. Bick, M. Goodfellow, C. R. Laing, and E. A. Martens: Understanding the dynamics of biological and neural oscillator networks through exact mean-field reductions: a review, J. Math. Neurosci. 10, 9 (2020).

[89] Y. Maistrenko, B. Lysyansky, C. Hauptmann, O. Burylko, and P. A. Tass: Multistability in the kuramoto model with synaptic plasticity, Phys. Rev. E 75, 066207 (2007).

[90] J. Ladenbauer, J. Lehnert, H. Rankoohi, T. Dahms, E. Schöll, and K. Obermayer: Adaptation controls synchrony and cluster states of coupled threshold-model neurons, Phys. Rev. E 88, 042713 (2013).

[91] S. Coombes and R. Thul: Synchrony in networks of coupled non-smooth dynamical systems: Extending the master stability function, Eur. J. Appl. Math. 27, 904 (2016).

[92] S. M. Crook, G. B. Ermentrout, M. C. Vanier, and J. M. Bower: The role of axonal delay in the synchronization of networks of coupled cortical oscillators, J. Comput. Neurosci 4, 161 (1997).

[93] R. A. Stefanescu and V. K. Jirsa: A low dimensional description of globally coupled heterogeneous neural networks of excitatory and inhibitory neurons, PLoS Comput Biol 4, e1000219 (2008).

[94] I. Omelchenko, O. E. Omel'chenko, P. Hövel, and E. Schöll: When nonlocal coupling between oscillators becomes stronger: patched synchrony or multichimera states, Phys. Rev. Lett. 110, 224101 (2013). 
[95] W. Gerstner, W. M. Kistler, R. Naud, and L. Paninski: Neuronal Dynamics: From single neurons to networks and models of cognition (Cambridge University Press, 2014).

[96] D. S. Bassett, P. Zurn, and J. I. Gold: On the nature and use of models in network neuroscience, Nat. Rev. Neurosci. 19, 566 (2018).

[97] J. Drover, J. Rubin, J. Su, and G. B. Ermentrout: Analysis of a canard mechanism by which excitatory synaptic coupling can synchronize neurons at low firing frequencies, SIAM J. Appl. Dyn. Syst. 65, 69 (2004).

[98] M. Wechselberger: Existence and bifurcation of canards in $\mathbb{R}^{3}$ in the case of a folded node, SIAM J. Appl. Dyn. Syst. 4, 101 (2005).

[99] X. Li, J. Wang, and W. Hu: Effects of chemical synapses on the enhancement of signal propagation in coupled neurons near the canard regime, Phys. Rev. E 76, 041902 (2007).

[100] W. J. Yuan and C. Zhou: Interplay between structure and dynamics in adaptive complex networks: Emergence and amplification of modularity by adaptive dynamics, Phys. Rev. E 84, 016116 (2011).

[101] E. Tang and D. S. Bassett: Colloquium: Control of dynamics in brain networks, Rev. Mod. Phys. 90, 031003
(2018).

[102] P. De Lellis, M. di Bernardo, F. Garofalo, and M. Porfiri: Evolution of complex networks via edge snapping, IEEE Trans. Circuits Syst. I 57, 2132 (2010).

[103] W. Yu, P. DeLellis, G. Chen, M. di Bernardo, and J. Kurths: Distributed adaptive control of synchronization in complex networks, IEEE Trans. Autom. Control 57, 2153 (2012).

[104] J. Lehnert, P. Hövel, A. A. Selivanov, A. L. Fradkov, and E. Schöll: Controlling cluster synchronization by adapting the topology, Phys. Rev. E 90, 042914 (2014).

[105] E. Schöll, S. H. L. Klapp, and P. Hövel: Control of selforganizing nonlinear systems (Springer, Berlin, 2016).

[106] See Supplemental Material at http... for the proof and details on the master stability function for adaptive networks of non-diffusively coupled oscillators, for details on the master stability function for the phase oscillator model, for the definition of the cluster parameter, for details on the desynchronization transition in the phase oscillator model, for details on the network model of coupled FitzHugh-Nagumo neurons with synaptic plasticity, and for details on the master stability function and the desynchronization transition in the model of adaptively coupled FitzHugh-Nagumo neurons. 